FAST CHANNEL SYNDROME: PRESENTATION, DIAGNOSIS AND MANAGEMENT IN AN INFANT
A. Mallappa ${ }^{1}$, S. Tirupathi², J. Boyd ${ }^{2}$, B. O 'Connor', M. Illingworth'

${ }^{1}$ Royal Belfast for Sick Children, ${ }^{2}$ Neurology, ${ }^{3}$ Paediatrics, Royal Belfast for Sick Children, Belfast, UK

Aims: To report a fast channel congenital myasthenic syndrome in an infant who presented as a floppy baby with episodes of acute apnoeas.

Methodology: Case notes from a tertiary hospital were reviewed and additional literature search was performed.

Report: A 9 week old infant was referred to a tertiary unit from a local district general hospital with a history of floppiness, recurrent feeding difficulties and intermittent apnoeas. The apnoeic episodes were associated with bradycardias and desaturations needing bag and mask ventilation on most occasions.

He had had ENT, speech and language assessment, MRI brain and a basic metabolic workup at the time of transfer.

In our hospital he was further assessed by various professionals and investigated accordingly for a floppy baby syndrome.

At 4 months of age he was admitted to paediatric intensive care unit following a respiratory arrest. On this occasion was as noted to have ptosis and opthalmoplegia which was more obvious $\mathrm{He}$ had a Tensilon test performed which was positive (video clip available). Following this he had nerve conduction tests and electro myography which confirmed a myasthenic syndrome.

DNA testing revealed the diagnosis of a fast channel congenital myasthenic syndrome.

He has responded well to pyridostigmine, but continues to have infrequent intermittent respiratory crises. Management issues are discussed.

Conclusion: This is the first case of fast channel congenital myasthenic syndrome in Northern Ireland. Ptosis and opthalmoplegia may not be obvious on initial examination, all floppy infants should be explored for congenital myasthenic syndrome.

\section{THE FIRST TURKISH CASE OF GLUCOSE TRANSPORTER TYPE 1 DEFICIENCY SYNDROME (GLUT 1D) WITH MOLECULAR STUDIES}

\author{
H. Gumus ${ }^{1}$, A.O. Caglayan², H. Per ${ }^{1}$, \\ S. Kumandas ${ }^{1}$, K. Engelstad ${ }^{3}$, F. Kardes ${ }^{1}$, \\ D. De Vivo ${ }^{3}$ \\ ${ }^{1}$ Erciyes University, ${ }^{2}$ Kayseri Education and \\ Research Hospital, Kayseri, Turkey, ${ }^{3}$ Columbia \\ University, New York, NY, USA
}

Glucose transporter type1 (GLUT-1) deficiency syndrome (OMIM 606777) is a autosomal dominantly inherited complex neurologic disorder that causes severe learning difficulties, ataxia, seizures, acquired microcephaly and developmental delay. We present a first Turkish case with GLUT1-Deficiency syndrome which is confirmed with molecular studies and additional four cases described. Children presenting with a clinical phenotype consisting of a refractory seizure disorder, ataxia and developmental delay should prompt the consideration of Glucose transporter 1 deficiency syndrome.

663

\section{DEMOGRAPHICS AND DIAGNOSES - A PAEDIATRIC NEURODEVELOPMENTAL CLINIC'S EXPERIENCE}

S. Bolton ${ }^{1}$, D. McDonald ${ }^{1}$, E. Curtis ${ }^{1}$, L. Gallagher ${ }^{2}$

${ }^{1}$ Department of Community Paediatrics, ${ }^{2}$ Department of Child Psychiatry, Adelaide and Meath Incorporating the National Children's Hospital (AMNCH), Tallaght, Dublin, Ireland

Background and aims: Children from ethnic minorities are likely to receive a diagnosis of autism 1.4 years later than non minority children and are at increased risk of developmental delay. This study aims to identify referral patterns, diagnoses, family developmental history, and nationality presenting to a paediatric neurodevelopmental clinic.

Methods: A review of medical records of children referred to a paediatric neurodevelopmental clinic from 2007 to 2009 was performed.

Results: 342 children presented. 218 (64.17\%) were Irish and 101 (29.5\%) were from a minority ethnic group. Of this group 54 (53.5\%) were African. The ethnicity of $23(6.7 \%)$ was unknown. 118 children $(34.5 \%)$ had a primary diagnosis of 